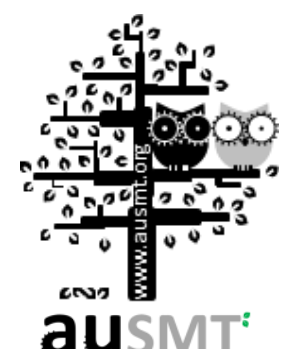

\title{
Modeling and Control of Twin Parallel-Axis Linear Servo Mechanisms for High-Speed Machine Tools
}

\author{
Wu-Sung Yao, Fu-Yun Yang, and Mi-Ching Tsai * \\ Department of Mechanical Engineering, National Cheng Kung University, Taiwan \\ (Received 31 December 2010; Accepted 29 April 2011; Published online 1 September 2011) \\ *Corresponding author: mctsai@mail.ncku.edu.tw \\ DOI: 10.5875 /ausmt.v1i1.72
}

Abstract: This paper presents a synchronous control scheme for a twin parallel-axis linear servo mechanism with mechanical coupling which appears inherently in the so-called box-in-box configuration of machine tools. To identify the two-port mathematical model, a multi-variable identification method is developed to construct a dynamic model of the controlled servo mechanism. Moreover, a synchronous controller framework based on the identified model is proposed to reduce position and velocity errors of the two coupled linear servomotors. To demonstrate the capacity of the mathematical model as well as the effectiveness of the control scheme, a gantry-type platform was utilized as an illustrated example, where the steady-state synchronization error reaches $0.5 \mathrm{~m}$ with a velocity of $830 \mathrm{~mm} / \mathrm{s}$.

Keywords: dynamic modeling; synchronous control; linear servomotor; system identification.

\section{Introduction}

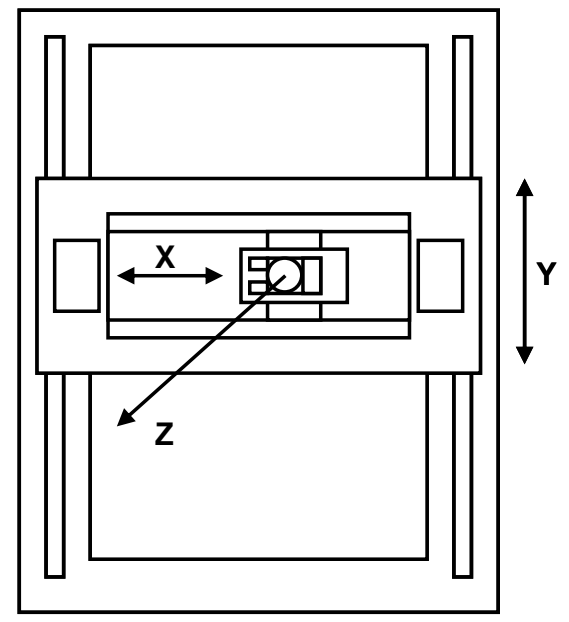

Figure 1. Box-in-box structure.

Synchronization motion control of multi-axis machine tools has become a significant issue due to the growing demand for high speed precise manufacturing. For such an application, two direct feed-drive linear servomotors are often adopted and arranged into a twin-parallel structure, the so-called "box-in-box" layout, to increase the thrust and stiffness of machine tools.
The box-in-box structure adopts a pair of linear servomotors with mechanical coupling of one feed axis. Figure 1 shows an example in which the $Y$-axis is driven by the two linear motors coupled with the $X$-axis feed drive. An important requirement for high speed precision machining is the capability of the twin-parallel feed drives to follow the same command trajectories accurately for achieving a satisfactory positioning accuracy and also for reducing the synchronization error of the two parallel motors.

Strategies of the synchronous motion control technique can be classified into three categories: (1) synchronous master motion control, (2) master-slave motion control, and (3) relative dynamic stiffness motion control [1]. The framework of Category (1) is depicted in Figure 2, which is also called parallel synchronous control. As illustrated in Figure 2, the two servomotors receive the same motion command simultaneously and are controlled independently without considering the motion of the other. The advantage of this control scheme lies in its simple structure. However, accurate synchronous movement may not be guaranteed due to some possible effects such as unknown disturbances or unmatched model. This scheme is not suitable for the box-in-box machine tools since inherent disturbances 
always exist due to inevitable nonlinear dynamics, such as mechanical coupling. Note that asynchronous operation in motion control may result in diminished machining accuracy of the work-piece or even lead to breakage of the device.

To improve this shortcoming, Sarachik and Ragazzini proposed the master-slave control scheme of Category (2) and applied it to a biaxial system [2]. In comparison to the synchronous master motion control, the master-slave control operates in a cascade manner, enabling the slave servo system to follow the master with higher dynamic stiffness. Thus, the framework of master-slave control provides an alternative solution which allots a great deal of attention to synchronous motion control. As shown in Figure 3, a tandem control of two servo systems with velocity feedforward compensation was proposed by FANUC Ltd. to drive a single axis of motion [3]. Furthermore, another master-slave control based on speed/torque coupling was developed by SIEMENS [4].

The synchronous error can be eliminated in the master-slave framework if the disturbance occurs in the master. However, the servo lag between the master axis and the slave axis causes synchronization errors, while the unidirectional communication renders the master axis unable to detect the asynchronous movement when the slave axis encounters uncertain load disturbances. Therefore, the so-called relative dynamic stiffness motion control of Category (3) was proposed in which the synchronous error can be compensated by both axes. Figure 4 illustrates a position/velocity hybrid

Wu-Sung Yao was born in Taiwan in 1972. He received the M.S. and Ph.D. degrees in the Department of Mechanical Engineering (ME) at National Cheng Kung University (NCKU), Tainan, Taiwan in 1997 and 2002, respectively. He is currently a Postdoctoral Researcher at the Department of ME at NCKU. His research interests include motor control engineering, piezoelectric engineering, advance control theory, and digital signal processing engineering.

Fu-Yun Yang was born in Taiwan in 1981. He received his B.S. degree in mechanical engineering from National Cheng Kung University (NCKU), Tainan, Taiwan, in 2003, and M.S. degree in mechanical engineering from Nationa Taiwan University, Taipei, Taiwan, in 2005. He is currently a Ph.D. candidate in the Department of Mechanical Engineering at NCKU. His research interests include motor control engineering, advance control theory, and digital signal processing engineering.

Mi-Ching Tsai was born in Taiwan, in 1956. He received the B.S. and M.S degrees in electronic engineering from National Taiwan University of Science and Technology, Taiwan, in 1981 and 1983, respectively, and the Ph.D. degree from the Department of Engineering Science, University of Oxford, Oxford, U.K., in 1990. In 1996, he became a Full Professor in the Department of Mechanical Engineering, National Cheng Kung University, Tainan, Taiwan From 2003 to 2004, he was also a Visiting Scholar in the Control Division, Department of Engineering, Cambridge University, Cambridge, U.K. From 2007 to 2010, he was appointed as the Director General of the Department of Engineering and Applied Sciences, National Science Council, Taiwan. He is currently a Director General at the Research and Services Headquarters and a Chair Professor with the Department of Mechanical Engineering at National Cheng Kung University. He is the author or coauthor of more than 90 published refereed journal papers, and he is a holder of more than 50 patents. His research interests include robust control, servo control, motor design, and applications of advanced control technologies using DSPs.

Copyright (C) 2011 International Journal of Automation and Smart Technology synchronous control proposed by Hsieh [5] with the concept of relative dynamic stiffness motion control. The same idea is also adopted by Yang [6] for controlling dual parallel linear servomotors. The key feature of relative dynamic stiffness motion control is that two motors compensate each axis in the velocity loop, but the position controller is only employed on the master. This framework can eliminate the servo lag error which occurs in the master-slave framework, while inharmonious movement in velocity loops can be compensated for in each axis. For further discussion of these three categories, one can refer to [1]. This paper utilizes relative dynamic stiffness motion control as the control strategy due to the benefits described above.

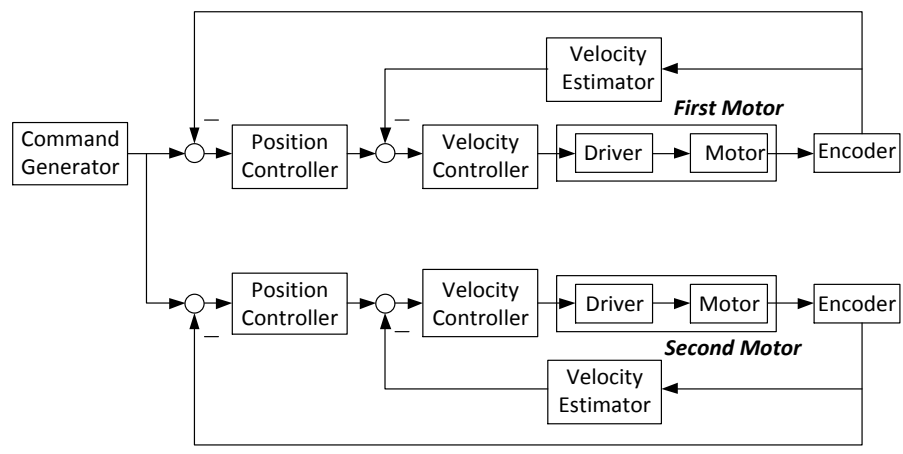

Figure 2. Parallel synchronous control.

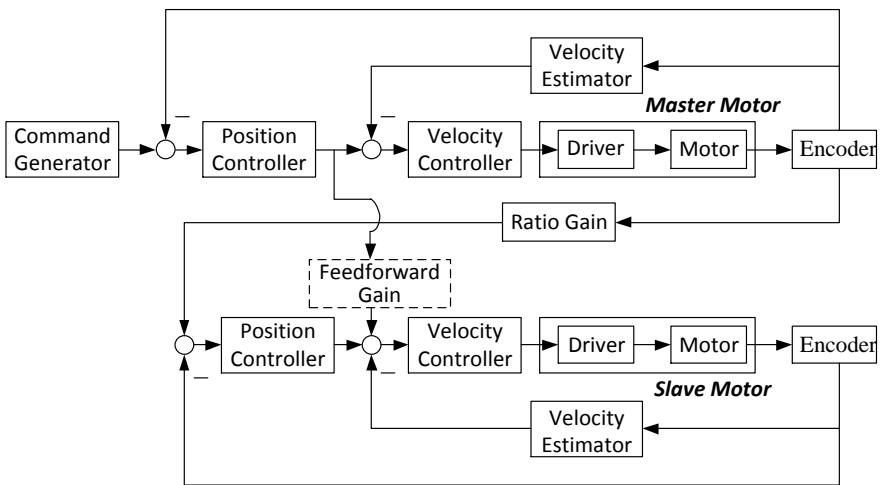

Figure 3. Tandem control with velocity feedforward compensation [7].

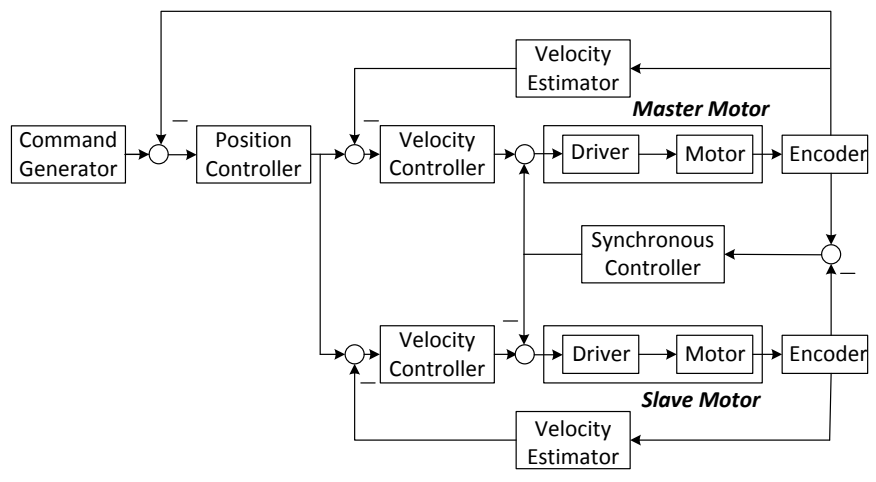

Figure 4. Relative dynamic stiffness motion control of [5] 
Much previous research has focused on the velocity synchronization problem of two-axis motion. Recently, great progress has been made in utilizing the cross-coupling concept to solve position synchronization of multiple motion axes. Chiu and Tomizuka provided a necessary coupling effect, which was introduced to each axis by the proper choice of a Lyapunov-like function [7]. In addition to actual position and velocity information, the resulting control law requires prior knowledge of the desired trajectories and their time derivatives. Sun proposed a new control framework for position synchronization of multiple motion axes by incorporating cross-coupling technology into the adaptive control architecture [8]. The above-mentioned approaches are commonly applied to multi-axis motion control systems rather than configurations, such as the "box-in-box" system.

In the "box-in-box" servo mechanism, an accurate model of the controlled plant is useful for the design of the synchronous control, particularly in the presence of mechanical coupling. Therefore, this paper presents an identification method to identify the effect of mechanical coupling. Figure 5 shows a gantry-type machine tool which is driven directly by three linear servomotors in which a pair of motors forms the $\mathrm{X}$-axis coupling with the $Y$-axis. An identification method is employed to characterize the dynamics of the mechanical coupling and a synchronous control scheme is also developed for the $\mathrm{X}$-axis to achieve synchronized motion control of the coupled systems. For clarification and simplicity, the error of motion between these two parallel servo systems in the $\mathrm{Y}$-axis is hereafter defined as the "synchronization error", which is the target to be eliminated by the proposed controller. It is noted that non-zero synchronization error causes the coupling to bend and generates force acting on both driving motors.

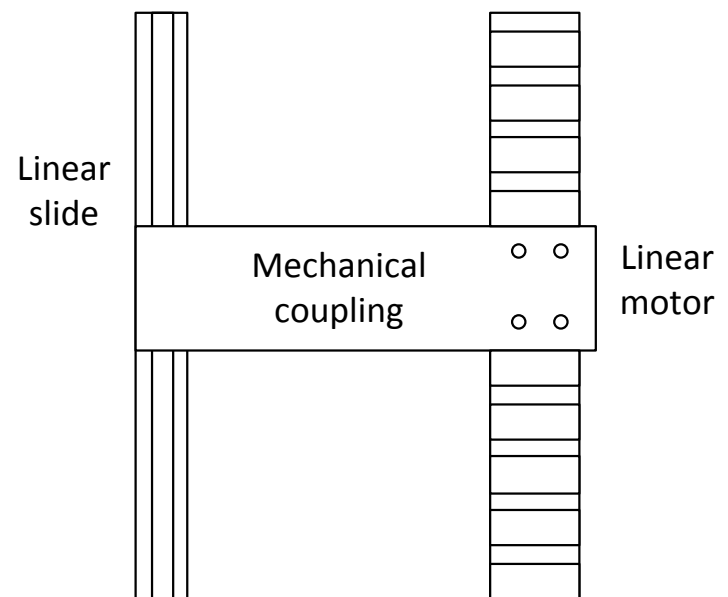

Figure 5. Gantry-type machine driven by a linear motor with a linear guide way.

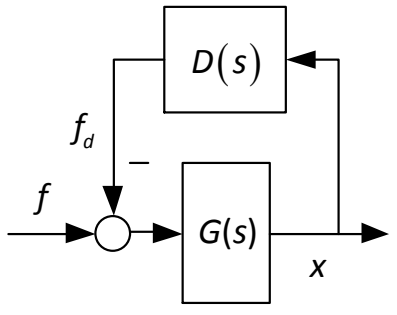

Figure 6. System model from the thrust command to the output.

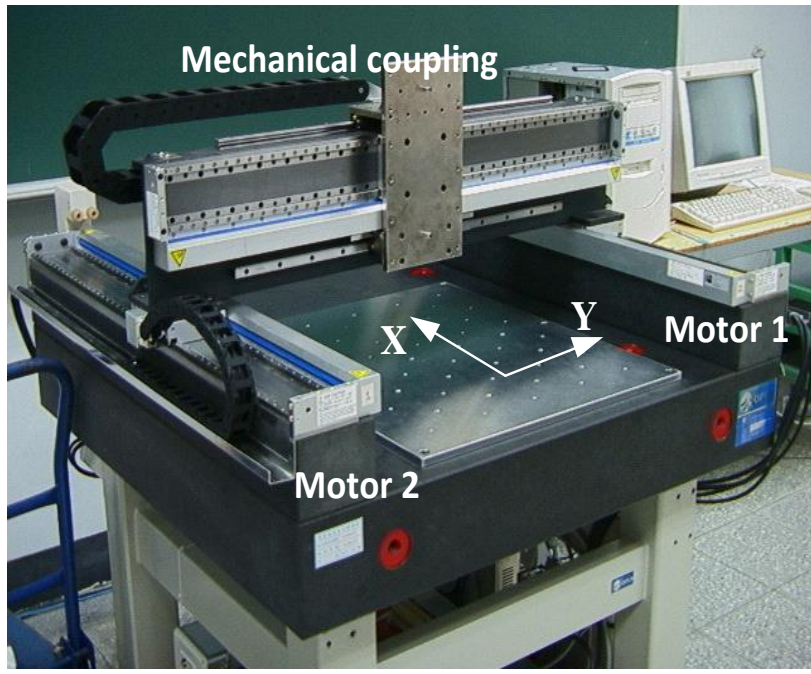

Figure 7. The linear motor driven machine tool.

\section{System Modeling}

\section{Modeling of Mechanical Coupling System}

Consider a gantry-type machine as shown in Figure 5 , where the working platform is driven by a linear servo motor with a linear guide way in an axis direction. As the un-synchronous behavior occurs between the dragged axis and the linear motor, a dragged force takes place on the linear motor due to the mechanical coupling between the motor and linear guide. Let $G$ denotes the mathematical model of the linear motor, $f$ the trust command, $f_{d}$ the external force driven by the mechanical coupling, and $D(s)$ represents the equivalent coupling effect of the mechanism. The control diagram of this system can be represented by Figure 6 .

Furthermore, take the synchronous control problem of the twin-parallel linear servomechanism with mechanical coupling into consideration. As illustrated in Figure 7 , the controlled plant is the two parallel linear servomotors in the $\mathrm{X}$-axis with the mechanical coupling and the velocity of each motor measured by an optical encoder. The working area of the machine tool is approximately $500 \mathrm{~mm} \times 500 \mathrm{~mm}$. The main specifications of the linear motors are listed in Table 1. 
Table 1. Specification of the linear motor.

\begin{tabular}{|l|l|}
\hline Max. thrust $(\mathrm{N})$ & 100 \\
\hline Max. speed $(\mathrm{mm} / \mathrm{s})$ & 830 \\
\hline Encoder resolution $(\mu \mathrm{m})$ & 0.5 \\
\hline Max. load weight $(\mathrm{N})$ & 200 \\
\hline
\end{tabular}

For the non-synchronous behavior of the twin-parallel motors, the force due to deformation of mechanical coupling (denoted "deformation force" hereafter) can be determined by the synchronization errors (position and velocity) between the two motors. It is noted that the material of the mechanical coupling is assumed to be homogenous and the deformation is sufficiently small. Therefore, the nonlinear coupling effects can be approximated by linear models, while the free body diagrams of the coupled mechanism can be illustrated by Figure 8 . As illustrated in this figure, the forces acting on each unit consist of the input thrust ( $T_{1}$ and $T_{2}$ converted from motor torque) and the deformation force $T_{d}$. Then, the deformation force $T_{d}$, due to any non-synchronization of these units, is given as $T_{d}=C(s) \cdot\left(V_{1}-V_{2}\right)$, where $C(s)$ represents the dynamic behavior of the mechanical coupling. It is noted that the deformation force results in drag thrust applied to the master motor as well as assistance thrust in the slave motor.

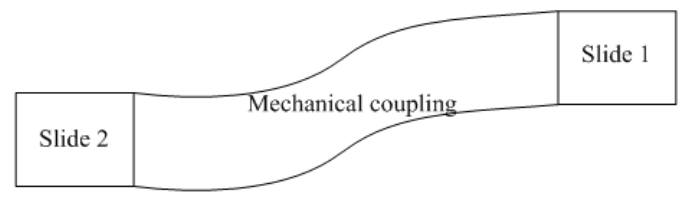

(a)

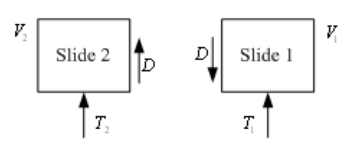

(b)

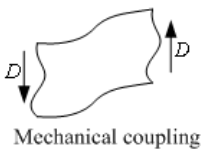

(c)
Figure 8. Free body diagrams: (a) mechanical deformation due to non-synchronization occurs; (b) forces on slide 1/slide 2, and (c) deformation forces on mechanical coupling.

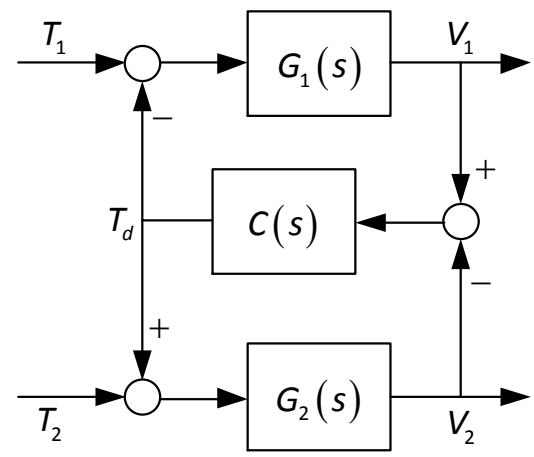

Figure 9. System model with the mechanical coupling.
For convenience, the two linear servomotors are denoted as Motor 1 and Motor 2, respectively. A dynamic model of the controlled plant (i.e., the two driving motors with coupling) in the twin-parallel linear servomechanism is shown in Figure 9, where $G_{1}(s)$ represents the transfer function for the thrust input $T_{1}$ to the velocity output $V_{1}$ of Motor 1 , while $G_{2}(s)$ is that of Motor 2. Note that the parameters with subscript "1" (or "2") are hereafter associated with Motor 1 (or Motor 2). The force $T_{d}$ caused by the deformation of the mechanical coupling is due to non-synchronization motion. For the case of $T_{2}=0$, one has, from Figure 9 ,

$$
\frac{V_{1}}{T_{1}}=\frac{G_{1}\left(1+C G_{2}\right)}{1+C G_{1}+C G_{2}}
$$

and

$$
\frac{V_{2}}{T_{1}}=\frac{C G_{1} G_{2}}{1+C G_{1}+C G_{2}}
$$

Similarly, for the case of $T_{1}=0$,

$$
\frac{V_{1}}{T_{2}}=\frac{C G_{1} G_{2}}{1+C G_{1}+C G_{2}}
$$

and

$$
\frac{V_{2}}{T_{2}}=\frac{G_{2}\left(1+C G_{1}\right)}{1+C G_{1}+C G_{2}}
$$

The system identification here is of practical interest. This paper employs the principle of superposition to determine the system model. Let the block diagram of Figure 9 be represented equivalently in matrix form as

$$
\left[\begin{array}{l}
V_{1} \\
V_{2}
\end{array}\right]=\left[\begin{array}{ll}
P_{11} & P_{12} \\
P_{21} & P_{22}
\end{array}\right]\left[\begin{array}{l}
T_{1} \\
T_{2}
\end{array}\right],
$$

where

$$
P_{11}=\frac{G_{1}\left(1+C G_{2}\right)}{1+C G_{1}+C G_{2}}, \quad P_{12}=P_{21}=\frac{C G_{1} G_{2}}{1+C G_{1}+C G_{2}},
$$

and

$$
P_{22}=\frac{G_{2}\left(1+C G_{1}\right)}{1+C G_{1}+C G_{2}} .
$$

Note that $P_{11}(s)$ and $P_{22}(s)$ denote the transfer functions from $T_{1}$ to $V_{1}$ and $T_{2}$ to $V_{2}$, respectively, and $P_{12}(s)$ the transfer function from $T_{2}$ to $V_{1}$, which characterizes the effect of the mechanical coupling, while $P_{21}(s)$ represents the transfer function from $T_{1}$ to $V_{2}$. Thus, the coupling dynamics $c(s)$ illustrated in Figure 9 are incorporated by $P_{12}(s)$ and $P_{21}(s)$. 


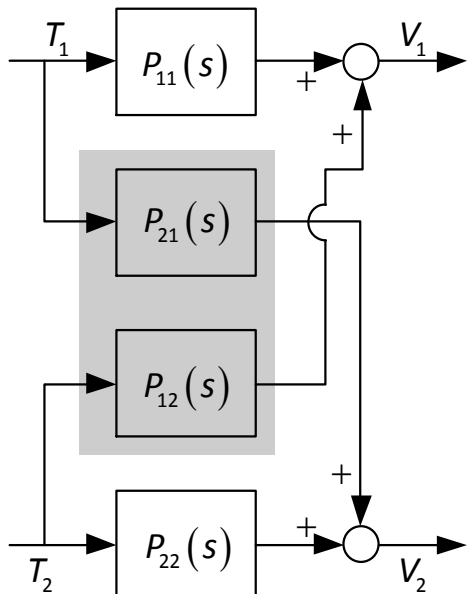

Figure 10. Control system composed of four open-loop transfer functions

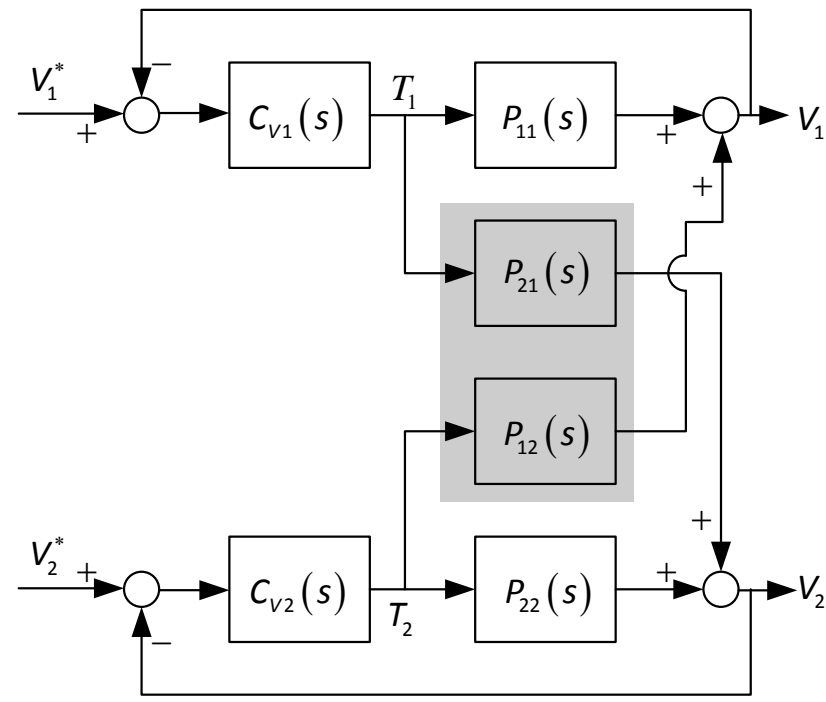

Figure 11 . The control system with velocity controllers

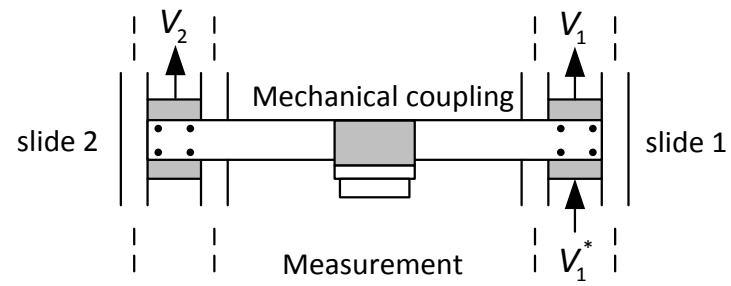

$V_{1}^{*}$ : velocity input of motor 1

$V_{1}$ : velocity output of motor 1

(a)

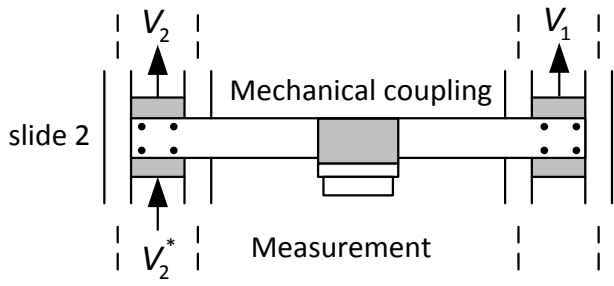

$V_{2}^{*}$ : velocity input of motor 2

$V_{2}$ : velocity output of motor 2 slide 1

Figure 12. Multi-variable system identification
The system diagram shown in Figure 10 represents the equivalent model of the coupled twin-parallel linear servo mechanism in an open-loop structure. Although the system model in (5) can be measured directly by open-loop identification methods, the closed-loop identification shown in Figure 11 is adopted to achieve better accuracy under disturbances. In the closed loop scheme of Figure 11, $C_{V 1}(s)$ and $C_{V 2}(s)$ refer to the velocity controllers of Motor 1 and Motor 2, respectively. The frequency response of the closed-loop system from velocity command input to the measured output can be used to determine the coupled system of Figure 10.

Figure 12 illustrates a practical identification process of the investigated system, in which the actuated motor drags the free slides (e.g., slide 2 in Figure 12(a) and slide 1 in Figure 12(b)) via the mechanical coupling in the system identification. First, Motor 1 (slide 1 ) is fed with a swept sine velocity command $V_{1}^{*}$ to drive the twin-parallel system, including the two slides and the mechanical coupling, while Motor 2 keeps free movement so that slide 2 is passively dragged to move by Motor 1 via the coupling, as illustrated in Figure 12(a). By measuring the corresponding velocity outputs of Motor 1 and Motor 2, i.e., $V_{1}$ and $V_{2}$, the transfer functions from $V_{1}^{*}$ to $V_{1}$ and $V_{1}^{*}$ to $V_{2}$ can be determined by the measured frequency responses. Repeating the same procedure, as shown in Figure 12(b), Motor 2 is actuated while slide 1 maintains free movement so that the closed-loop transfer functions from $V_{2}^{*}$ to $V_{2}$ and $V_{2}^{*}$ to $V_{1}$ can be obtained. The closed-loop identification results are depicted below, where the velocity controllers are set to $C_{V 1}=1.9$ and $C_{V 2}=1.6$. Therefore, the transfer functions in (5) can be solved via decoupling the equations of (6) to (9).

$$
\begin{aligned}
& T_{V_{1}^{*}-V_{1}}(s)=\frac{1110.1}{s^{2}+27.96 s+1110.1} \\
& T_{V_{2}^{*}-V_{2}}(s)=\frac{1365.9}{s^{2}+27.9 s+1365.9}
\end{aligned}
$$

$$
T_{V_{1}^{*} V_{2}}(s)=\frac{38149}{s^{3}+57.43 s^{2}+1758.8 s+38149}
$$

$$
T_{V_{2}^{*}-V_{1}}(s)=\frac{38618}{s^{3}+55.34 s^{2}+1689.28 s+38618}
$$




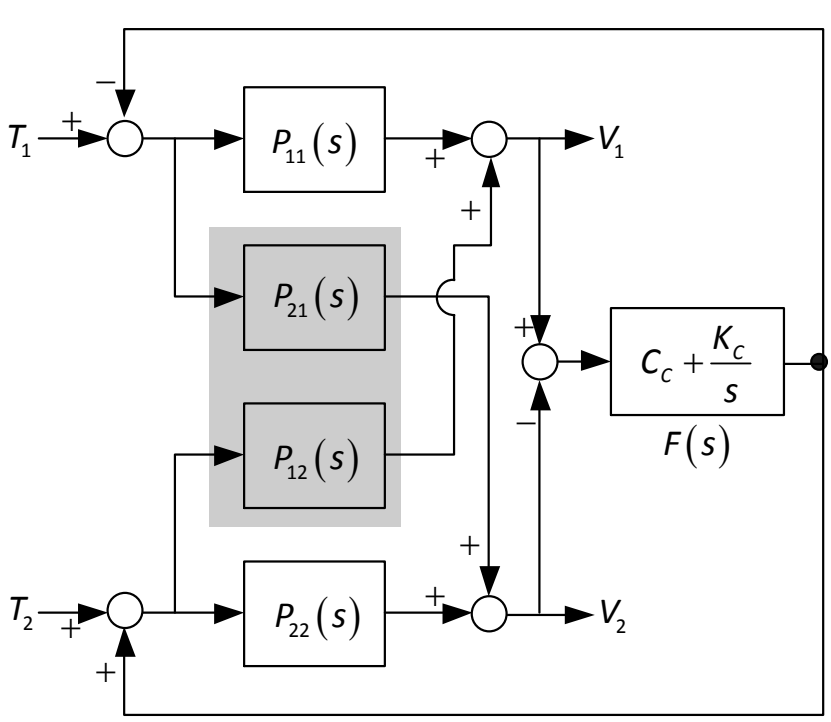

Figure 13. Synchronous compensator.

\section{Synchronous Controller Design}

Based on the multi-variable model of Figure 10, the synchronous compensator $F(s)=\left(C_{c} s+K_{c}\right) / s$ shown in Figure 13 was designed to compensate thrust such that the deformation of the mechanical coupling and the resulting force due to non-synchronization in the twin-parallel servo mechanisms can be reduced. For convenience, let $E=V_{1}-V_{2}$ denote the synchronization error. Then one has

$$
\begin{aligned}
& V_{1}=\frac{\left(P_{11}+P_{11} \cdot F \cdot P_{22}-F \cdot P_{12} \cdot P_{21}\right)}{1+F \cdot H} T_{1} \\
& +\frac{\left(P_{12}-F \cdot P_{12} \cdot P_{21}+P_{11} \cdot F \cdot P_{22}\right)}{1+F \cdot H} T_{2}
\end{aligned}
$$

$$
\begin{aligned}
& V_{2}=\frac{\left(P_{21}-F \cdot P_{12} \cdot P_{21}+P_{11} \cdot F \cdot P_{22}\right)}{1+F \cdot H} T_{1} \\
& +\frac{\left(P_{22}+P_{11} \cdot F \cdot P_{22}-F \cdot P_{12} \cdot P_{21}\right)}{1+F \cdot H} T_{2}
\end{aligned}
$$

where $H=P_{11}-P_{21}+P_{22}-P_{12}$. The synchronization error can be derived by

$$
E=\frac{\left(P_{11}-P_{21}\right) T_{1}+\left(P_{12}-P_{22}\right) T_{2}}{1+F \cdot H}
$$

For comparison, the synchronization error $E_{0}$ for the case without the synchronous compensator (i.e., $F(s)=0$ ) in Figure 13 is given by

$$
E_{0}=\left(P_{11}-P_{21}\right) T_{1}+\left(P_{12}-P_{22}\right) T_{2}
$$

Comparing (12) and (13) yields

$$
E=\frac{E_{0}}{1+F \cdot H}
$$

Therefore, the magnitude of the synchronization error can be reduced if $F(s)$ is chosen such that

$$
\sup \left\{\frac{1}{1+F \cdot H}\right\} \leq 1, \forall \omega \leq \omega_{c}
$$

then

$$
|E|=\left|\frac{E_{0}}{1+F \cdot H}\right| \leq\left|E_{0}\right| \cdot\left|\frac{1}{1+F \cdot H}\right|<\left|E_{0}\right|
$$

where $\omega_{c}$ denotes the desired bandwidth.

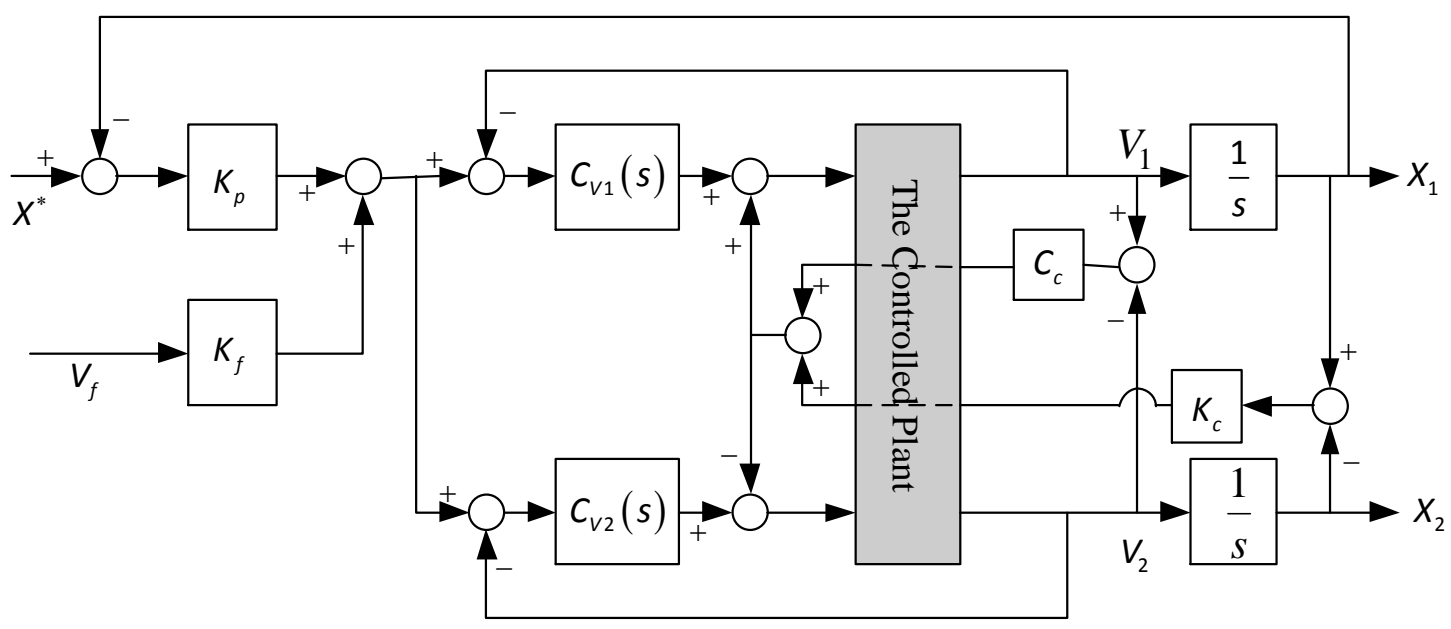

Figure 14. The proposed synchronous control structure. 


\section{Experimental Studies}

Based on the result of the illustrated example, the synchronous control scheme shown in Figure 14, namely hybrid stiffness/compliance synchronous control, was investigated where the gray box represents the controlled plant, and the position loop controller $K_{p}$ and velocity feedforward compensation $K_{f}$ are employed to achieve a better transient response. This scheme adopts compliance control in that Motor 1 is for position control, and Motor 2 is compliant with the velocity command of Motor 1 . Given the velocity loop controllers $C_{V 1}=1.9+0.002 / \mathrm{s}$ and $C_{V 2}=1.6+0.002 / \mathrm{s}$, the synchronous compensator $F(s)$ to speed up convergence of the synchronization error was determined as

$F(s)=\frac{0.2 s+0.8}{s}$ (i.e., $C_{c}=0.2$ and $K_{c}=0.8$ ).

Figure 15 illustrates the magnitude plots of $1 /(1+F H)$. With the design rule of (14), it can be concluded that the compensator $F(s)$ does work while the system operates within the bandwidth of $\omega_{c}=48 \mathrm{rad} / \mathrm{s}$.

The position loop gain and the feedforward gain were determined to be $K_{p}=2$ and $K_{f}=0.3$, respectively, to yield a satisfactory transient response. The control algorithms were implemented by a DSP (digital signal processor) controller (TMS320C32) for real-time computations, where the sampling time was $10 \mathrm{~ms}$. Note that the maximum thrust of $100 \mathrm{~N}$ is generated at the $8 \mathrm{~V}$ command.

In the experimental configuration, two desired trajectories with S-curve planning were considered. For case 1 , the parameters for the command are the position $r=500 \mathrm{~mm}$, the maximum velocity $v_{\max }=500 \mathrm{~mm} / \mathrm{s}$, the maximum acceleration $a_{\max }=2800 \mathrm{~mm} / \mathrm{s}^{2}$, and the average acceleration $a_{\text {ave }}=1600 \mathrm{~mm} / \mathrm{s}^{2}$, as shown in Figure 16. The position responses of the master and slave motors are shown in Figures 17 and 18 respectively. The measured synchronization error of the twin-parallel linear servo mechanism is shown in Figure 19. In case 2, the maximum velocity $v_{\max }$ is set to be $870 \mathrm{~mm} / \mathrm{s}$, while other parameters remain unchanged, as shown in Figure 20. The position responses of the master and slave motors are shown in Figures 21 and 23 respectively, while the synchronization error response is shown in Figure 22. Clearly, the transient synchronization error of case $1(500 \mathrm{~mm} / \mathrm{s})$ is lower than that of case $2(870 \mathrm{~mm} / \mathrm{s})$, while the steady-state reaches an encoder resolution of $0.5 \mu \mathrm{m}$. Note that the synchronization error, as previously mentioned, is the error between the two parallel motors instead of the positioning errors.

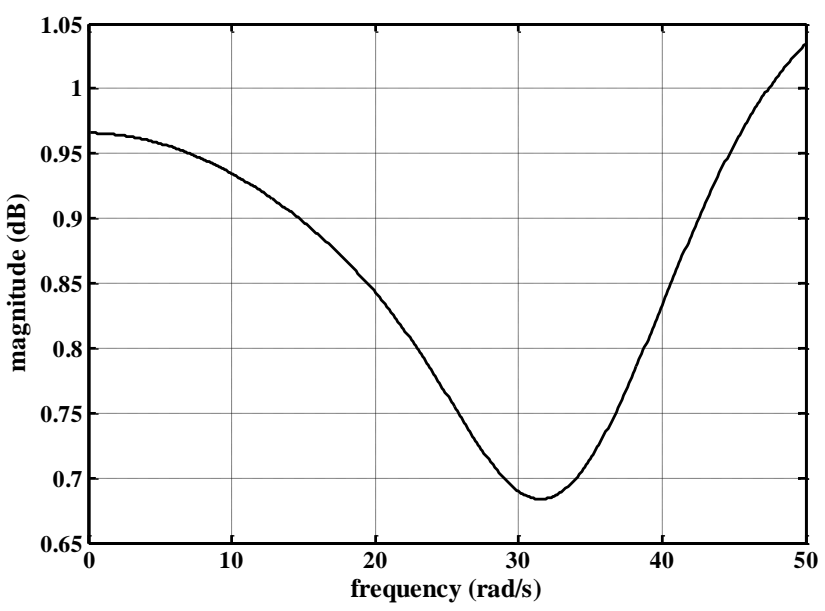

Figure 15. The magnitude plot of $1 /(1+F H)$

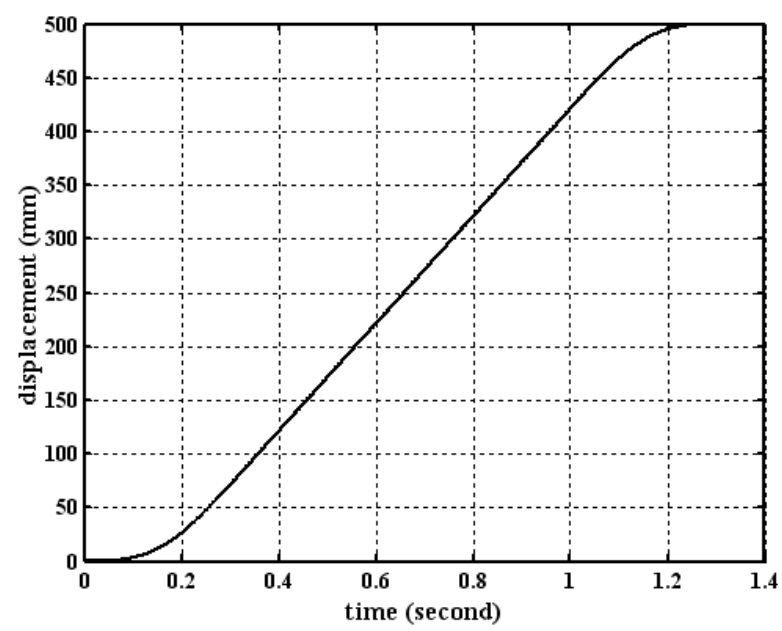

Figure 16. The desired trajectory with S-curve planning in case 1.

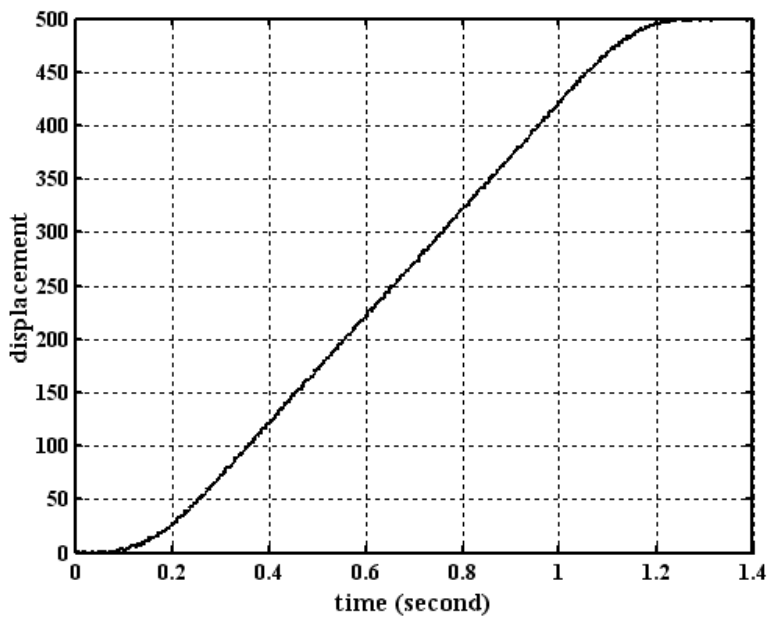

Figure 17. Position response of Motor 1 in case 1. 


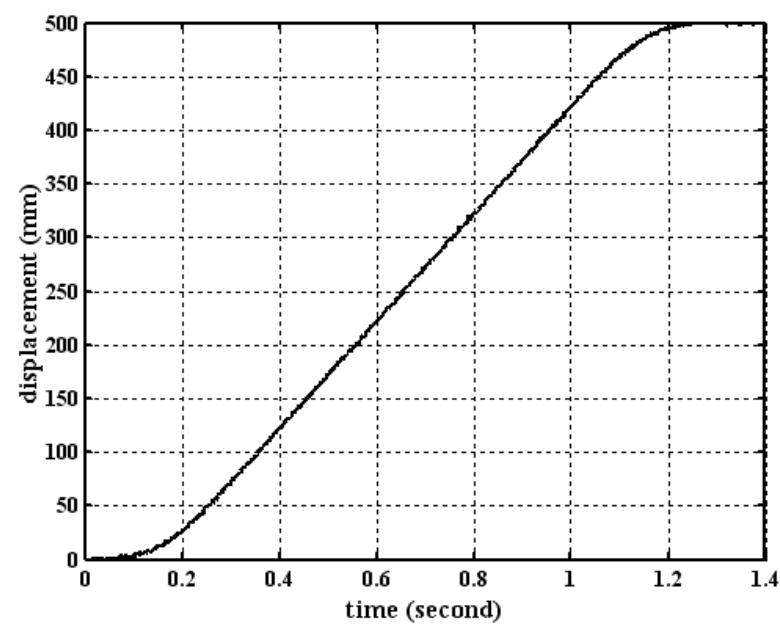

Figure 18. Position response of Motor 2 in case 1.

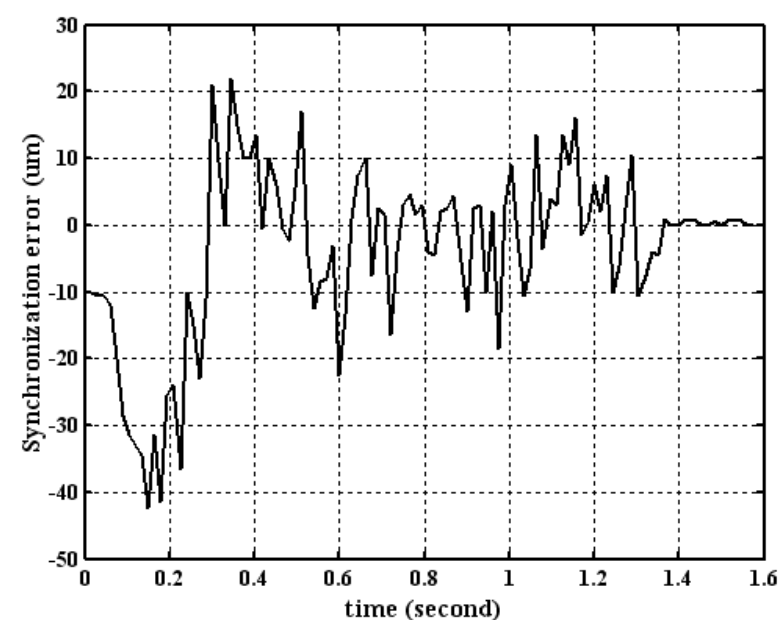

Figure 19. The synchronization error response in case 1.

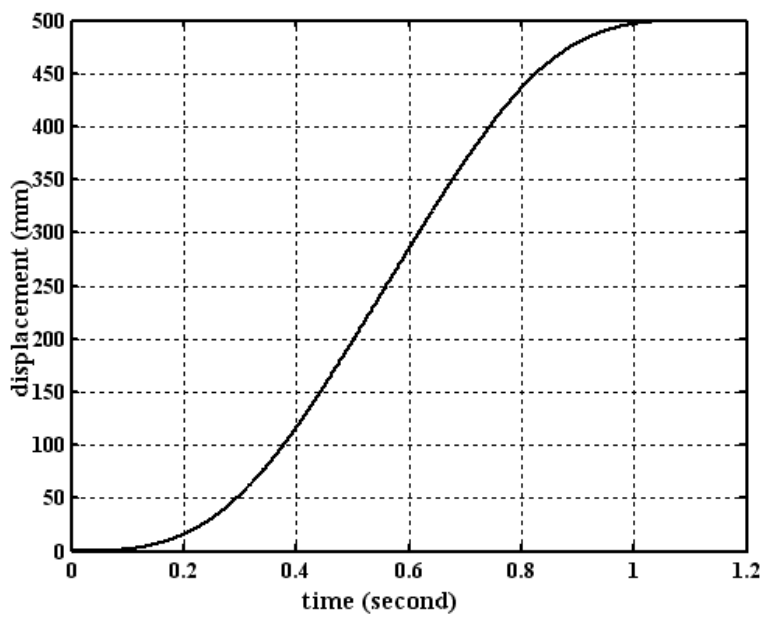

Figure 20. The desired trajectory with S-curve planning in case 2.

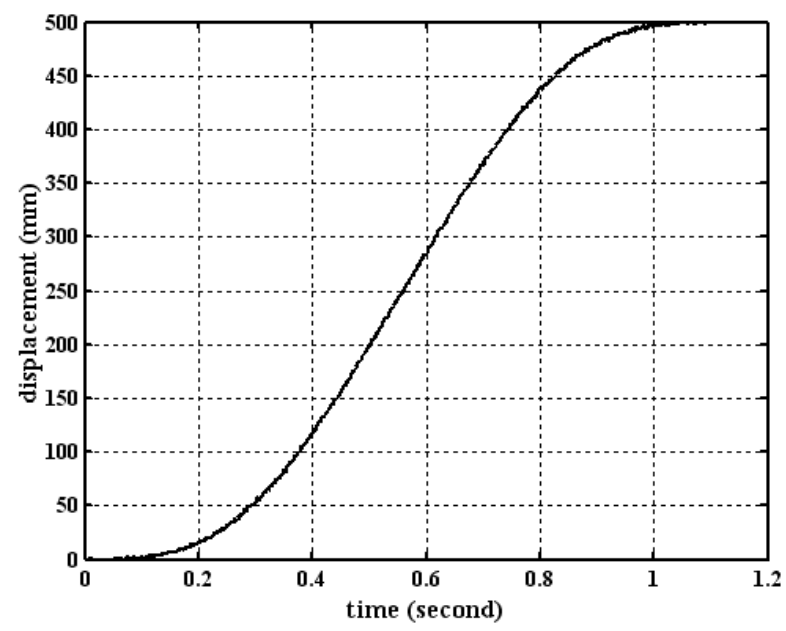

Figure 21. Position response of Motor 1 in case 2.

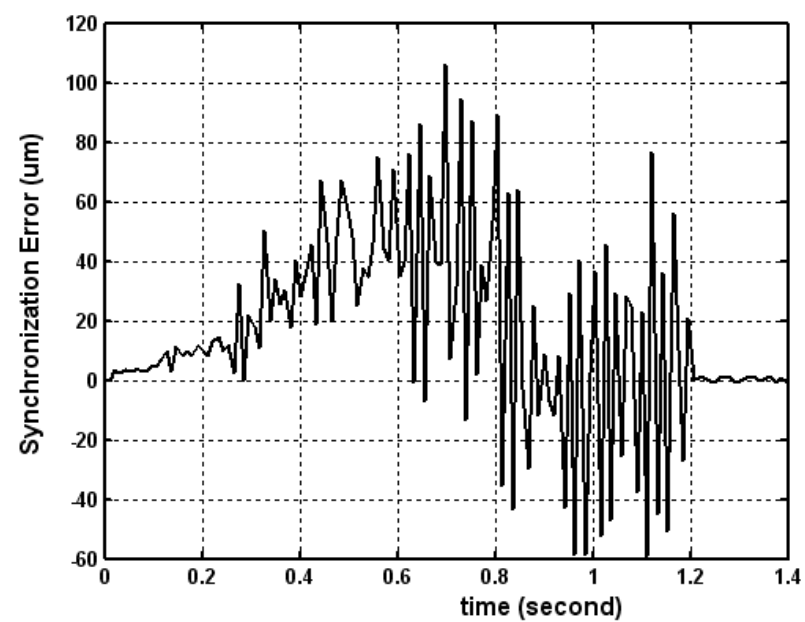

Figure 22. The synchronization error response in case 2.

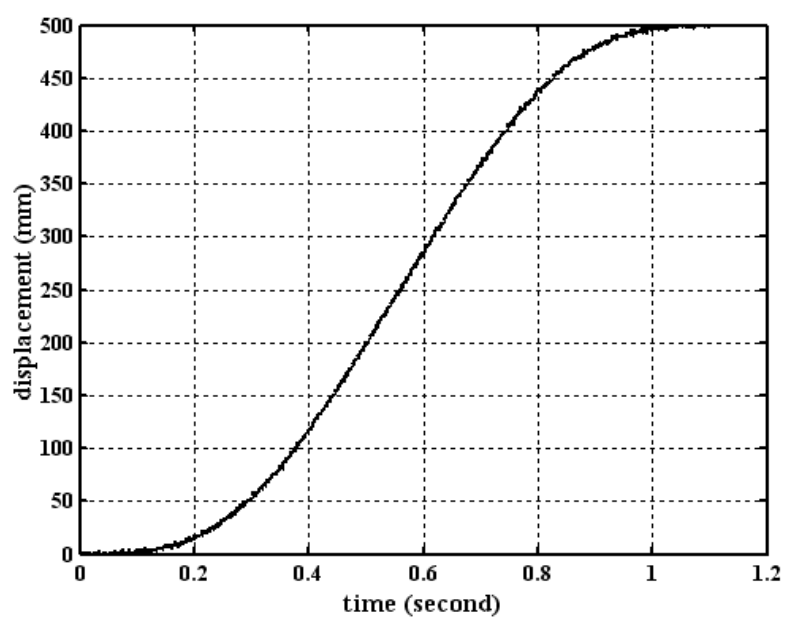

Figure 23. Position response of Motor 2 in case 2. 


\section{Conclusion}

This paper has developed a two-port system modeling technique for the axis control of a high-speed gantry-type machine tool with mechanical coupling. Based on the two-port model, a stiffness/compliance synchronous control scheme with a deformation force compensator was proposed to reduce the synchronous error of the machine tool. The effectiveness of the modeling and control in minimizing the synchronization error were validated by the experimental data. It can be concluded that synchronous control of high speed machining with mechanical coupling can be achieved by the proposed system modeling and control technique.

\section{References}

[1] R. D. Lorenz and P. B. Schmidt, "Synchronized motion control for process automation," Industry Applications Society Annual Meeting, San Diego, CA , USA, 1989, pp. 1693-1698. doi: 10.1109/IAS.1989.96869

[2] P. Sarachik and R. J.R., "A 2-dimensional feedback control system," Transactions of the American Institute of Electrical Engineers, vol. 76, part 2, pp. 55-61, 19571957.

[3] FANCU AC servo motor Alpha-series, Parameter Manual, FANCU Corp., 1994.

[4] 840D/FM-NC Description of functions, special functions (Part 3), SIEMENS,1999.

[5] M. F. Hsieh, C. J. Tung, W. S. Yao, M. C. Wu, and Y. S. Liao, "Servo design of a vertical axis drive using dual linear motors for high speed electric discharge machining," International Journal of Machine Tools and Manufacture, vol. 47, no. 3-4, pp. 546-554, 2007.

doi: 10.1016/j.ijmachtools.2006.05.011

[6] J. X. Yang, "The identification and control of a twin linear servo system with mechanical coupling," M.S. thesis, Department of Mechanical Engineering, National Cheng Kung University, Taiwan, 2003.

[7] G. T. C. Chiu and M. Tomizuka, "Coordinated position control of multi-axis mechanical systems," Journal of Dynamic Systems, Measurement, and Control, vol. 120, no. 3, pp. 389-393, 1998. doi: $10.1115 / 1.2805413$

[8] D. Sun, "Position synchronization of multiple motion axes with adaptive coupling control," Automatica, vol. 39, no. 6, pp. 997-1005, 2003. doi: 10.1016/S0005-1098(03)00037-2
[9] M. F. Hsieh, W. S. Yao, and C. R. Chiang, "Modeling and synchronous control of a single-axis stage driven by dual mechanically-coupled parallel ball screws," The International Journal of Advanced Manufacturing Technology, vol. 34, no. 9-10, pp. 933-943, 2007. doi: 10.1007/s00170-007-1135-4

[10] Y. Koren, "Cross-coupled biaxial computer control for manufacturing systems," Journal of Dynamic Systems, Measurement, and Control, vol. 102, no. 4, pp. 265-272, 1980. doi: $10.1115 / 1.3149612$

[11] Y. Koren and C. C. Lo, "Advanced controllers for feed drives," CIRP Annals - Manufacturing Technology, vol. 41, no. 2, pp. 689-698, 1992. doi: $10.1016 /$ S0007-8506(07)63255-7

[12] M. G. M. Lammers, "Linears lead in ultrasmooth motion," Machine design, vol. 66, no. 17, pp. 60-64, 1994.

[13] K. Srinivasan and P. K. Kulkarni, "Cross-coupled control of biaxial feed drive servomechanisms," Journal of Dynamic Systems, Measurement, and Control, vol. 112, no. 2, pp. 225-232, 1990. doi: $10.1115 / 1.2896129$

[14] M. Tomizuka, J. S. Hu, T. C. Chiu, and T. Kamano, "Synchronization of two motion control axes under adaptive feedforward control," Journal of Dynamic Systems, Measurement, and Control, vol. 114, no. 2, pp. 196-203, 1992 doi: $10.1115 / 1.2896515$

[15] X. C. Xi, A. N. Poo, G. S. Hong, and F. Huo, "Experimental implementation of taylor series expansion error compensation on a bi-axial cnc machine," The International Journal of Advanced Manufacturing Technology, no. 2010. doi: $10.1007 / \mathrm{s} 00170-010-2843-8$

[16] W. S. Yao, "Design of linear servo systems for high speed machine tools," Ph.D. dissertation, Department of Mechanical Engineering, National Cheng Kung University, Taiwan, 2002.

[17] D. Zhao, S. Li, F. Gao, and Q. Zhu, "Robust adaptive terminal sliding mode-based synchronised position control for multiple motion axes systems," IET Control Theory \& Applications, vol. 3, no. 1, pp. 136-150, 2009. doi: $10.1049 /$ iet-cta:20070272 